Ann. Zootech., I967, 16 (3), 255-262

\title{
NOTE SUR LA VALEUR COMPARÉE DE 4 MÉTHODES PERMETTANT D'ESTIMER L'IMPOR'TANCE DES MORCEAUX GRAS DANS UNE DÉCOUPE DE PORC
}

\author{
I. OLIIVIER et J. VANDERIIAEGEN \\ avec la collaboration technique de F. Aguillon, D. Brault, \\ C. Filatre, N. Gaudin, R. Marlot, M. Renault et A. Ridard \\ Station centrale de Génétique animale, \\ Centre national de Recherches zootechnique, 78-Jouy-en-Josas, \\ Institut national de la Recherche agronomique \\ Institut technique du Porc, 60, rue Caumartin, Paris - ge
}

SOMMAIRE

Quatre méthodes de mesure du gras dorsal ont été comparées sur un échantillon de 146 porcs pesant environ Ioo $\mathrm{kg}$ : les ultrasons sur l'animal vivant, la réglette métallique, le sondage optique et la mesure directe sur la carcasse fendue. Ces quatre méthodes se classent dans l'ordre suivant quant à leur valeur pour estimer l'importance des morceaux gras dans la carcasse : sondage optique, réglette, mesure directe et ultrasons. Cette étude montre que l'utilisation du sondage ultra sonore, qui tend à se généraliser dans la sélection du porc, peut, dans certains cas, donner des résultats relativement imprécis, car cette méthode exige des opérateurs très avertis et un appareil techniquement très au point.

La proportion des morceaux gras d'une carcasse de porc est le facteur le plus important de sa "qualité ». Cependant, une découpe de carcasse n'est pas toujours possible, soit que l'animal considéré doive éventuellement servir à la reproduction, soit que le temps et les moyens nécessaires à une telle découpe fassent défaut. Dans le premier cas, on a recours à des mesures sur l'animal vivant, dans le second cas à des mesures sur la carcasse fendue, le caractère mesuré étant, dans les deux cas, l'épaisseur du dépôt gras dorsal. Parmi les méthodes de mesure sur le vivant, dont une revue détaillée a été faite par Dumost (I957), deux retiennent l'attention, du fait de leur commodité d'emploi : les ultrasons et la réglette métallique. Les mesures sur la carcasse se font à des niveaux très variables (DUMONT et GoRGE, I960) et il semble difficile d'établir le "meilleur » emplacement de mesure le long de la colonne 
vertébrale. Par contre, de nombreuses études ont établi avec certitude que des mesures effectuées latéralement sont supérieures à des mesures effectuées dans le plan de fente de la carcasse. Un système optique permet d'effectuer ces mesures latérales sans couper transversalement la carcasse. Ces considérations nous ont amené à effectuer la comparaison des quatre méthodes suivantes d'appréciation d'une carcasse : mesures sur le vivant aux ultrasons ou à la réglette d'une part, mesure sur la carcasse par sondage optique latéral ou par mesure directe au niveau de la fente de la carcasse d'autre part. Cependant, pour des raisons pratiques, les mesures à la réglette n'ont pas été faites sur l'animal vivant, mais sur la carcasse immédiatement après l'abattage.

I 46 porcs (99 femelles et 47 mâles castrés) ont été utilisés. Ces porcs proviennent de la station de mise à l'épreuve des verrats sur leur descendance située à Jouy-enJosas, et correspondent aux abattages de Io semaines successives (mai-juillet I965). Leur poids d'abattage varie de 98 à $105 \mathrm{~kg}$.

Chaque animal a subi successivement 4 séries de mesures :

I. I a veille de l'abattage : mesures avec un appareil à ultrasons (1) de l'épaisseur du lard en six emplacements situés symétriquement à $4 \mathrm{~cm}$ de part et d'autre de la ligne médiane, soient 2 mesures à l'arrière de l'épaule, 2 mesures au dos (au niveau de la dernière côte) et 2 mesures au rein (à environ $55 \mathrm{~cm}$ en avant de la queue) ;

2. Le jour de l'abattage, environ une demi-heure après l'abattage, mesure à l'aide d'une réglette métallique aux mêmes emplacements que ci-dessus, sur la carcasse suspendue.

3. Immédiatement après les mesures précédentes, mesure par sondage optique $\left({ }^{2}\right)$ des épaisseurs de lard à des emplacements situés à environ un demi-centimètre en avant ou en arrière des précédentes.

4. Le lendemain de l'abattage mesure directe des épaisseurs de lard à 3 niveaux: dernière vertèbre lombaire, dernière vertèbre dorsale et dernière vertèbre cervicale.

Des opérateurs différents ont effectué chacune des quatre séries de mesures.

De plus, le lendemain de l'abattage, la demi-carcasse droite de chaque porc a été découpée selon la méthode parisienne normalisée et le rapport du poids des morceaux gras (bardière et panne) au poids de la demi-carcasse découpée a été calculé.

Les données ont été analysées par la méthode de la régression multiple en considérant, d'une part deux variables dépendantes (ou prédites) : poids de la bardière dans la demi-carcasse et pourcentage de morceaux gras, et d'autre part quatre ensembles de quatre variables indépendantes (ou prédictrices) qui sont :

I. Poids vif, moyenne de deux mesures aux ultrasons au rein, au dos et à l'épaule.

2. Poids vif, moyenne de deux mesures à la réglette au rein, au dos et à l'épaule.

3. Poids de la demi-carcasse découpée et moyenne de deux mesures à l'endoscope. au rein, au dos et à l'épaule.

4. Poids de la demi-carcasse découpée et mesures directes au rein, au dos et au cou (une seule mesure à chaque niveau).

L'analyse a été faite séparément pour les deux sexes. Trois races étaient représentées, Large White, Danoise et Blanc de l'Onest. La première étant prépondérante

(1) "Pig grader "Smiths.

(2) Endoscope Foster. 
dans l'échantillon, il n'a pas été tenu compte du facteur " race ". 'l'ous les calculs ont été effectués par voie mécanographique à la Station centrale de Cénétique animale.

Les moyennes des 16 variables considérées figurent au tableau I. I,es résultats de l'analyse de régression multiple sont résumés dans les tableaux 2 et 3 , pour le poids de la bardière et le pourcentage de morceaux gras respectivement. I es valeurs de $t$ permettant de tester la signification de chaque coefficient de régression partielle figurent entre parenthèses.

Le tableau I montre que pour les mesures au rein et au dos l'accord est satisfaisant entre les 4 méthodes de mesure (compte tenu d'une certaine imprécision sur les emplacements de mesure). Par contre, à l'épaule des différences systématiques existent entre les 3 méthodes appliquées à ce niveau, les ultrasons donnant les valeurs les plus élevées et le sondage optique les valeurs les plus faibles.

TABI,EAU I

Moyennes

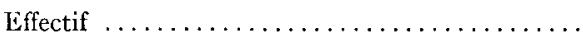

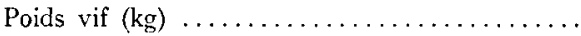

Poids de la demi-carcasse $(\mathrm{kg})$

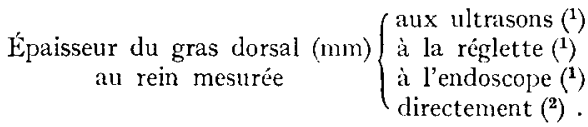

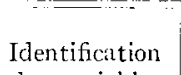
des variables

Epaisseur du gras dorsal (mm) $\left\{\begin{array}{l}\text { aux ultrasons }(1) \\ \text { à la réglette }\left({ }^{1}\right)\end{array}\right.$ au dos mesurée a lendoscope $(\mathbf{1})$
directement $\left({ }^{2}\right)$. Épaisseur du gras dorsal (mm)
$\dot{a}$ l'épaule mesurée $\left\{\begin{array}{l}\text { aux ultrisons }\left({ }^{1}\right) \\ \text { à la réglette }\left({ }^{1}\right) \\ \dot{a} \text { l'endoscope }\left({ }^{1}\right)\end{array}\right.$

Épaisseur du gras clorsal au cou (mm) mesurée directement $\left(^{2}\right) \ldots \ldots \ldots \ldots \ldots \ldots \ldots \ldots \ldots$ Poids de la bardière $(\mathrm{kg}) \ldots \ldots \ldots \ldots \ldots \ldots$ Pourcentage de morceaux gras (p. 100).

(1) Movenne de 2- mesures.

(2) Une seule mesure.

Le tableau 2 montre que les ensembles de mesures considérés peuvent expliquer de 26 à 74 p. Ioo de la variation totale du poids de la bardière. Le sondage opti(que est la méthode qui permet d'estimer le poids de la bardière avec la meilleure précision. La comparaison des 2 méthodes de mesure sur le vivant est nettement à l'avantage de la réglette qui, il faut cependant le souligner, a été utilisée sur la carcasse dans cette expérience. Les mesures à l'épaule effectuées aux ultrasons apparaissent peu précises. 
I. OLLIVIER, J. VANDERHAEGEN

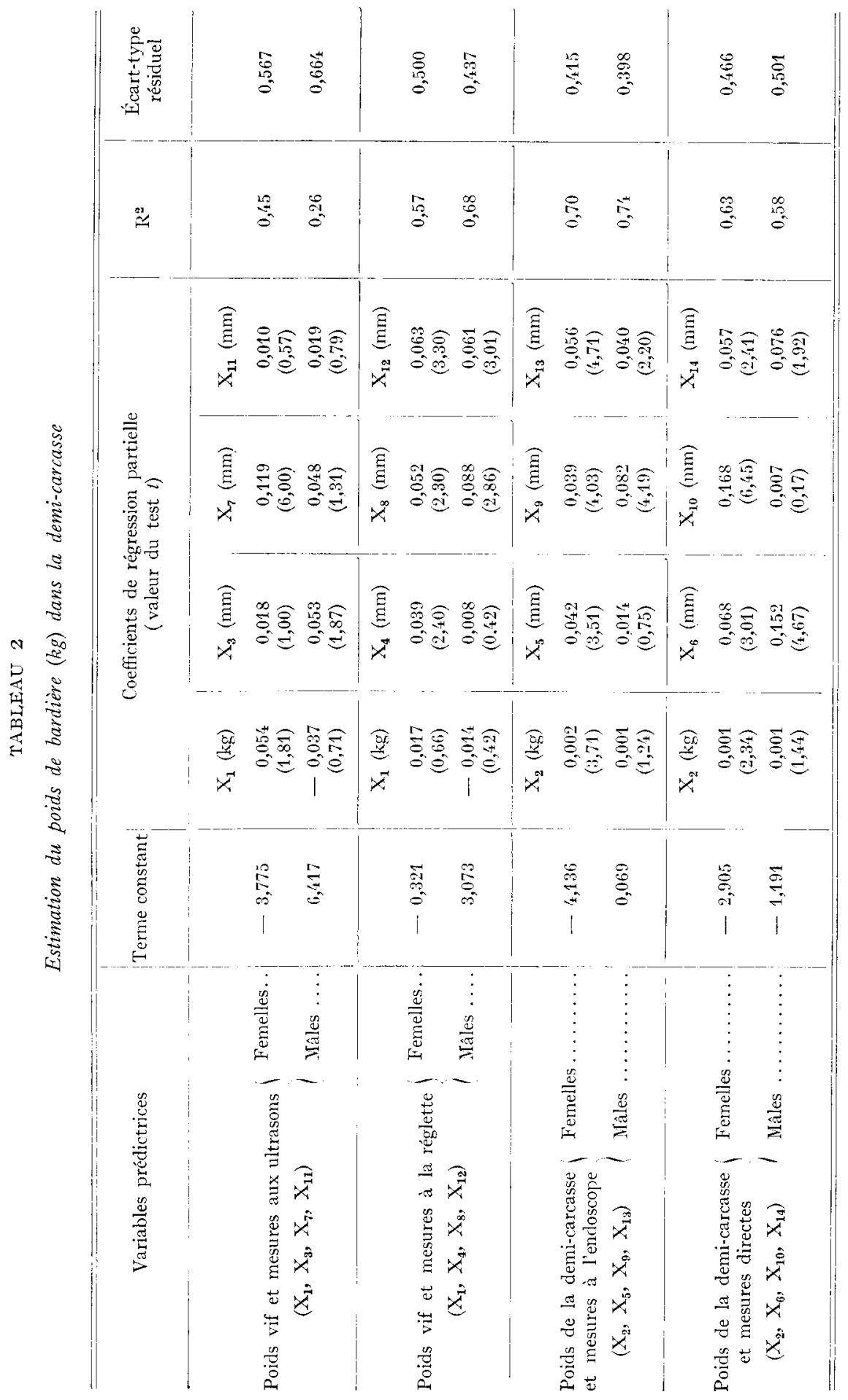


ESTIMATION DES MORCEAUX GRAS DU PORC

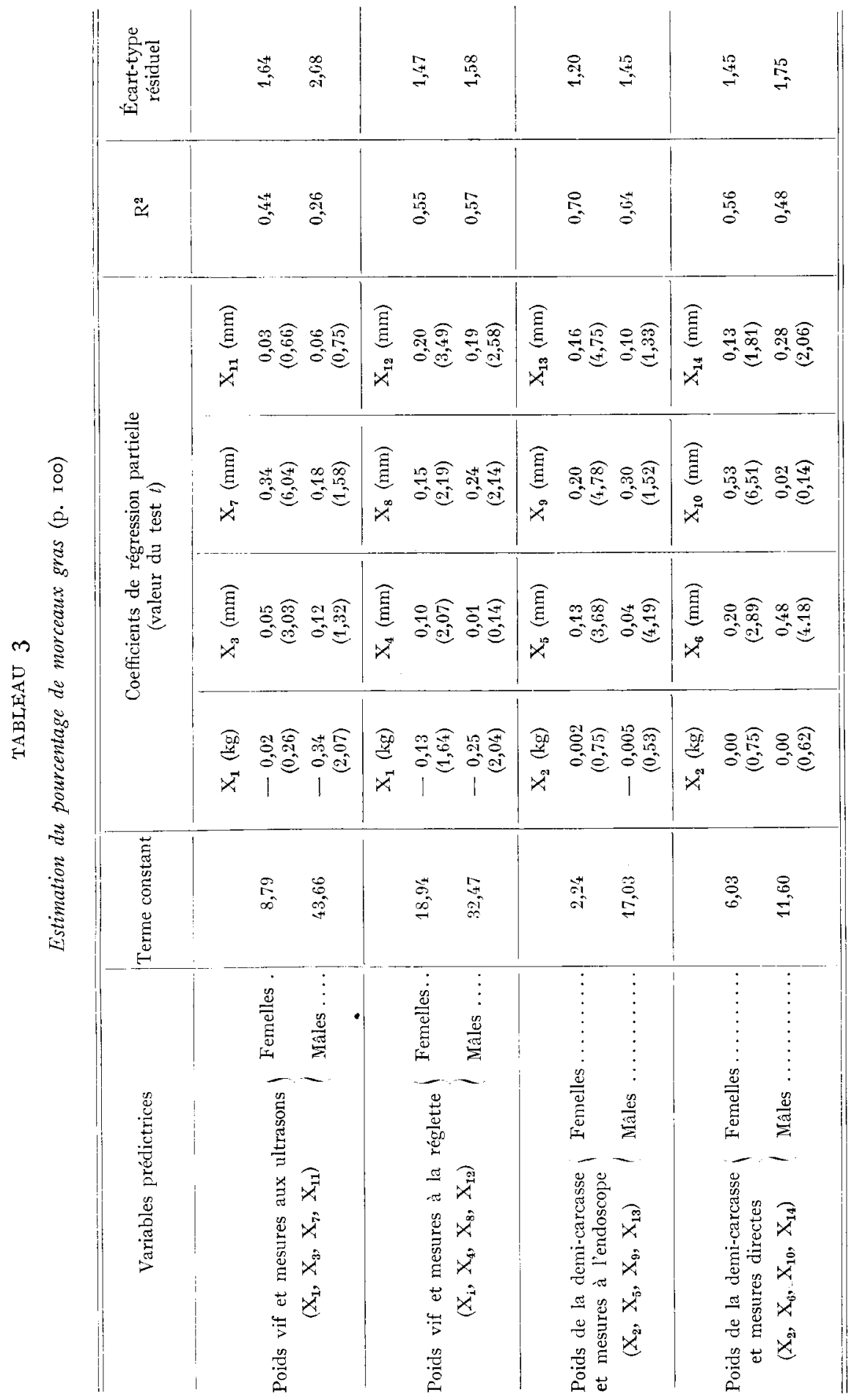


En effet, le test du coefficient de régression partielle relatif à ces mesures n'est pas significatif, alors qu'il l'est hautement pour les mesures à la réglette au même endroit. Le tableau 2 montre également que les mesures effectuées latéralement à l'endoscope sont meilleures que celles effectuées au niveau de la fente de la carcasse.

En ce qui concerne le pourcentage de morceaux gras (tabl. 3), les méthodes se classent de la même façon, la part de variation expliquée par ces mesures allant de 26 à $70 \mathrm{p}$. Ioo. Cette étude met en relief la faible précision relative de la méthode ultrasonore sur des porcs de roo $\mathrm{kg}$ et particulièrement sur les mâles castrés. L,es mesures au niveau de l'épaule sont les plus sujettes à erreur, particulièrement quand. l'opérateur, comme c'était le cas ici, ne possède pas une expérience suffisante pour "interpréter " la lecture des différents échos de l'oscilloscope. Les causes de cette imprécision ont été précédemment discutées (OLIIVIER, I965) et sont illustrées par la figure $\mathrm{I}$. I a comparaison des mesures aux ultrasons et à la réglette sur le même

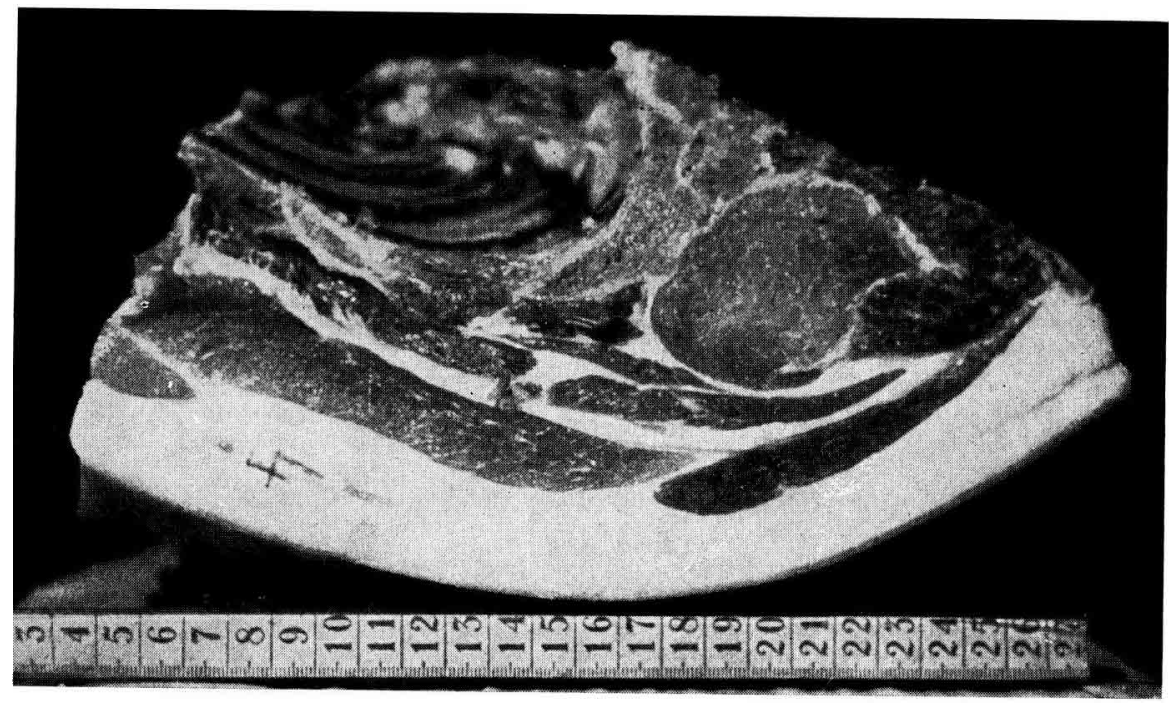

FIG. I. - Coupe transzersale d'une demi-carcasse droite au niveau des mesures à l'epante

animal est un moyen que l'on peut préconiser pour éviter à un "débutant " de grossières erreurs. Il est possible également que cette imprécision à l'épaule soit attribuable à l'appareil utilisé, l'image donné par l'oscilloscope se prêtant difficilement à interprétation. Nos résultats sont d'ailleurs en contradiction avec ceux de Dunon'T et DESTANDAU (I964) qui obtiennent avec un appareil à ultrasons une précision supérieure à celle que fournit la réglette, et diffèrent aussi de ceux de HAzEL et KIINE (I959) qui obtiennent avec les deux méthodes des précisions équivalentes.

Quant aux mesures sur la carcasse dans un but d'appréciation commerciale, il semble qu'un gain de précision important pourrait être obtenu en effectuant des mesures latérales qui ont l'avantage de n'être pas affectée par l'imprécision de la découpe. Ceci confirme les observations de plusieurs auteurs, en particulier Buck et al. (Ig62). Cependant des précautions sont à prendre dans l'utilisation de l'endoscope à 1'épaule. En effet, à ce niveau, la séparation entre les deux couches de lard 
est souvent marquée par un tissu conjonctif assez épais et coloré qu'il est difficile, avec cet appareil, de distinguer du muscle. La présence de ce conjonctif explique sans doute les faibles épaisseurs trouvées à l'épaule avec cette méthode.

Enfin cette étude confirme qu'une précision voisine de celle que permettent les mesures sur carcasse peut être obtenue sur l'animal vivant à la réglette, ce qui autorise une appréciation objective sur pied de la valeur commerciale de l'animal. L'utilisation de la réglette fait moins appel au "jugement " de l'opérateur que l'appareil à ultrasons, et, de ce fait, requiert un personnel moins expérimenté.

TABI,EAU 4

Accroissement du poids de bardière dans la demi-carcasse et du pourcentage de morceaux gras pour un accroissement uniforme de $1 \mathrm{~mm}$ d'épaisseur de lard

(à poids constant)

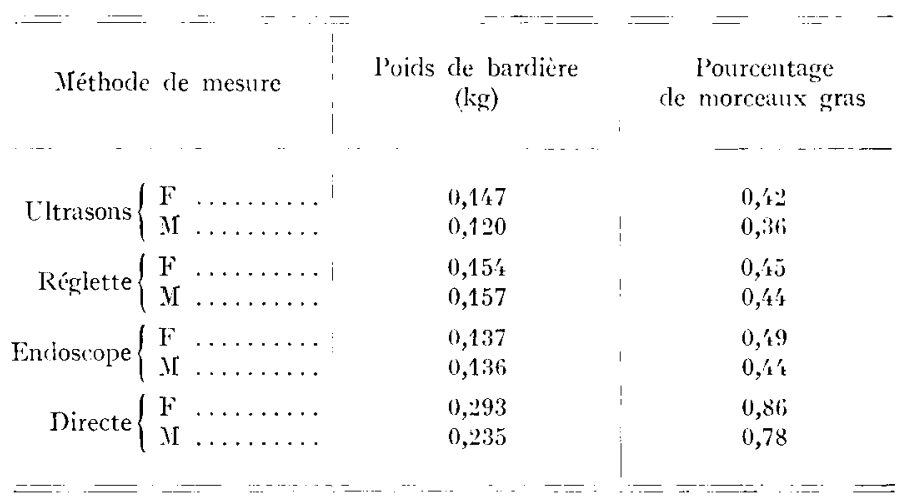

Le tableau 4 permet d'établir une équivalence approximative entre les 4 méthodes. Il apparait, en particulier, qu'une différence de $\mathrm{I}$ mm mesurée aux ultrasons ne correspond qu'à une différence moyenne de $0,5 \mathrm{~mm}$ environ sur la carcasse froide.

Ces résultats, cependant, ne s'appliquent rigoureusement qu'à des porcs dont le poids vif varie très peu autour de roo $\mathrm{kg}$ et une étude analogue serait à faire sur une gamme de poids plus étendue avant de pouvoir généraliser les conclusions auxquelles nous avons abouti.

Reçu pour publication en juin 1967.

\section{SUMMARY}

COMPARISON BETWEEN FOUR METHODS TOWARDS THE ESTIMATION OF THE PERCENT FAT CUTS IN PIGS CARCASSES

Four different methods of measuring back fat thickness were compared on 146 pigs of $100 \mathrm{~kg}$ average live-weight : ult rasonics on the live animal, metallic ruler, optical probe and direct measurement on the split carcass. These four methods rank in the followng order with respect to their value in the prediction of the percent fat cuts in the carcass : optical probe ; ruler ; direct measurement and ultrasonics.

It therefore appears that the ultrasonic technique, which is now widespread in pig selection, may, under certain conditions, give rather inaccurate results, for the method requires well-experienced operators and a technically adapted apparatus. 


\section{RÉFÉRENCES BIBLIOGRAPHIQUES}

Buck S. F., Harrington. F., Johnson R. F., ig62. The prediction of lean percentage of pigs of bacon weight from carcass measurements. Anim. Prod., 4, 25-36.

Dumont B. L., 1957. Nouvelles méthodes pour l'estimation de lá qualité des carcasses sur les porcs vivants. Réunion de la F.E.Z. sur l'épreuve de descendance des porcs., Copenhague.

Dumont B. L., Destandau S., ig64. Comparaison de quatre méthodes de mesure de l'épaisseur des tissus adipeux sous-cutanés chez le porc vivant. Ann. Zootech., 13, $213^{-2}$ i 6.

Dumont B. L., GoRGE C., I960. La signification de l'épaisseur du lard dorsal des porcs à différents niveaux de la colonne vertébrale. Réunion de la sous-commission porcinede la $l i, E$. Z. Letchworth (G. B.).

Hazel L. N., KLine E. A., 1959. Ultrasonic measurement of fatness in swine. J. Anim. Sci., 18, 81 5-819.

Oltivier L., I965. L'accroissement en épaisseur du tissu gras dorsal chez le verrat en croissance. Ann. Zootech., 14, 391-399. 